\title{
Foumal of Cancer
}

Research Paper

$2011 ; 2: 200-205$

\section{Medullary Thyroid Cancer: It is a pain in the neck?}

\author{
Marlon A. Guerrero ${ }^{1 凶}$, Sheila Lindsay ${ }^{2}$, Insoo Suh ${ }^{2}$, Menno R. Vriens ${ }^{2}$, Elham Khanafshar ${ }^{3}$, Wen T. Shen ${ }^{2}$, \\ Jessica Gosnell2, Electron Kebebew ${ }^{4}$, Quan-Yang Duh², Orlo H. Clark²
}

1. Department of Surgery, University of Arizona, Tucson, AZ, USA;

2. Department of Surgery, University of California, San Francisco, CA, USA;

3. Department of Pathology, University of California, San Francisco, CA, USA;

4. Surgery Branch, National Cancer Institute, Bethesda, MD, USA

\section{Corresponding author: Emails: mguerrero@surgery.arizona.edu}

(c) Ivyspring International Publisher. This is an open-access article distributed under the terms of the Creative Commons License (http://creativecommons.org/ licenses/by-nc-nd/3.0/). Reproduction is permitted for personal, noncommercial use, provided that the article is in whole, unmodified, and properly cited.

Received: 2010.10.28; Accepted: 2011.03.25; Published: 2011.04.08

\begin{abstract}
Background: Medullary thyroid cancer (MTC) commonly presents with lymph node (LN) metastases, and has a worse prognosis than papillary thyroid cancer (PTC). Tumor size and $\mathrm{LN}$ involvement have been shown to affect stage of disease; however, to our knowledge, ours is the first study that attempts to correlate anterior neck pain on presentation with the extent of disease.

Methods: We performed a retrospective review of patients with MTC who underwent an operation from February 1998 through December 2008. We compared the symptom of anterior neck pain with the pathologic extent of disease. Our control group comprised patients who underwent an operation for PTC. Analysis was performed using the Fisher's exact test and the Mann-Whitney test.

Results: Of the 109 patients with MTC, 50 (46\%) met our inclusion criteria. Of the 50 patients with MTC, II presented with neck pain, compared to 3 of the 50 patients with PTC (P $=0.04 \mathrm{I})$. Of those II patients, $9(82 \%)$ had LN involvement on final pathology, as compared with $14(36 \%)$ of the 39 without neck pain $(p=0.014)$. Of patients with neck pain, I8\% were diagnosed at stage I to II and $82 \%$ at stage III to IV, compared to $64 \%$ at stage I to II and $36 \%$ at stage III to IV ( $p=0.014)$.

Conclusions: Our study demonstrates that more patients with MTC present with anterior neck pain than do patients with PTC and that patients with MTC and neck pain have an increased risk of LN metastases. The results of this study suggest that MTC patients, who present with concomitant neck pain, should undergo a total thyroidectomy, prophylactic bilateral central neck dissection, and ipsilateral lateral neck dissection.
\end{abstract}

Key words: Medullary thyroid cancer, lymph node, papillary thyroid cancer

\section{Introduction}

Medullary thyroid cancer (MTC), which behaves more aggressively than well-differentiated thyroid cancer (WDTC) of follicular cell origin, accounts for $13 \%$ of all thyroid-related deaths.[1] Most cases of MTC arise sporadically, but about $25 \%$ are hereditary, as part of multiple endocrine neoplasia type 2 (MEN
$2 a$ and 2b) and isolated familial MTC (FMTC). Though both forms (i.e., sporadic and inherited) of MTC have a propensity for lymphatic and distant metastases,[2] the inherited form develops at an earlier age, is associated with C-cell hyperplasia, and is often multifocal. The differences in aggressive be- 
havior correlate with specific mutations in the rearranged during transfection (RET) protooncogene, mutations that are present in nearly all patients with inherited MTC. Similar somatic mutations are found in about $25 \%$ of patients with sporadic MTC.[3] The overall 10-year survival rate of patients with MTC is about $75 \%$, as compared with over $90 \%$ for patients with WDTC.[4-6]

Lymph node (LN) metastases are more common in patients with larger tumors, with specific RET mutations, and with markedly elevated calcitonin and carcinoembryonic antigen levels.[4] Clinical parameters also dictate the extent of disease; for example, palpable lateral LNs in patients with proven MTC are highly predictive of LN metastases. Other clinical features that suggest extensive disease include symptoms of dysphagia, dyspnea, and hoarseness. $[7,8]$ As such, the American Thyroid Association (ATA) recommends that all patients with sporadic MTC undergo a prophylactic central neck dissection during the initial total thyroidectomy.[5] However, a recent study reports that numerous surgeons are not following the new ATA guidelines.[9] Therefore, in order to support the recommendations for a central neck dissection in patients with MTC we sought to determine if a clinical parameter can predict the extent of disease in MTC.

It is intuitive to infer that anterior neck pain in patients with a palpable neck mass is associated with more extensive disease, but we sought to answer 2 questions, in particular, in our study: (a) Can the symptom of anterior neck pain predict the extent of disease in patients with no gross clinical features of thyroid cancer? (b) Are patients who complain of anterior neck pain and are found to have a thyroid nodule more likely to have MTC? Thus, the goals of our study were to determine whether or not the symptom of anterior neck pain occurred more frequently in patients with MTC than papillary thyroid cancer (PTC) and whether or not it predicted the clinical and pathologic extent of disease in patients with MTC.

\section{Materials and Methods}

We retrospectively analyzed the records of all patients who underwent a neck operation for MTC at the University of California, San Francisco, from February 1998 through December 2008. We defined a neck operation as a thyroidectomy alone, a thyroidectomy with central LN dissection, or a thyroidectomy with central and either ipsilateral or bilateral lateral LN dissection. We recorded patient characteristics, family history, preoperative ultrasound results, operative results, and pathology data. In addition, at the time of the initial preoperative encounter, each patient had prospectively completed a questionnaire addressing his or her presenting symptoms (e.g., neck pain, hoarseness, dysphagia, dyspnea). Neck pain was defined as any subjective complaint of anterior neck discomfort, ache, pressure, or sharp, throbbing, or dull sensations in the region of the thyroid gland, with or without palpation.

The inclusion criteria for our study included the diagnosis of newly diagnosed MTC (sporadic and inherited). We excluded patients who did not have documentation on the presence or absence of neck pain, either on the clinic evaluation or the questionnaire. Our control group consisted of randomly selected patients with PTC who underwent a thyroidectomy, with or without LN dissection during. The number of randomly selected PTC patients equaled the final number of patients in the MTC study group. The same exclusion criteria used for our study group was followed. The institutional review board at the University of California, San Francisco, approved the study.

We compared the presence or absence of neck pain with the clinical and pathologic extent of disease in patients with MTC and PTC. We defined the clinical extent of disease by the presence of palpable or ultrasound-proven LN metastasis and/or by evidence of wide metastatic disease. We also evaluated the extent of disease per the patient's final pathology test results, looking at capsular invasion, lymphatic invasion, vascular invasion, and LN involvement.

Data are reported as the mean \pm standard deviation (SD) or as numbers (percent). To compare the symptom of pain and the extent of disease, we used the Fisher's exact test; to compare nonparametric data, the Mann-Whitney test. For each variable, we calculated a 2-tailed p-value. We considered a p-value < 0.05 to be statistically significant.

\section{Results}

During our 130-month study period, 109 patients with MTC underwent a neck operation at our institution; $50(46 \%)$ met our inclusion criteria for evaluation. Our control group consisted of 50 randomly chosen patients who underwent a neck operation for PTC. We found no significant difference in age or race between patients the MTC and PTC patients, but there was a significant gender difference between both groups. Although women made up the majority of patients in both groups, there were disproportionally more women in the PTC control group than in the MTC study group (Table 1). Most patients in both groups were asymptomatic, but $22 \%$ of those with MTC had neck pain at presentation, as compared with only $6 \%$ of those with PTC $(\mathrm{p}=0.041)$. Only patients 
with MTC (2 of 50) had hoarseness; however, the difference between the 2 groups regarding this symptom was not statistically significant.

The final pathology test results regarding multifocality, capsular invasion, and vascular invasion did not significantly differ between the 2 groups. However, patients with MTC had a higher rate $(20 \%)$ of lymphatic invasion than those with PTC (2\%); this difference was significant $(p=0.008)$. Despite this increased risk of lymphatic invasion for MTC patients, their incidence of LN metastases was not higher than the incidence for PTC patients (Table 1).

The stage of disease at presentation significantly differed between the 2 groups. Patients with MTC presented less often with stage I disease $(32 \%)$, as compared with patients with PTC $(72 \%)(p=0.0001)$. Conversely, patients with MTC presented more often with stage IV disease (30\%), as compared with patients with PTC $(2 \%)(p=0.0001)$. We found no difference between the 2 groups in terms of stage II and stage III at presentation.

Of the 50 patients with MTC, 11 (22\%) had neck pain. Neck pain was distributed in the region of the thyroid gland, and no patient presented with neck pain from palpable lymphadenopathy. No patient with MTC presented with clinically palpable lymphadenopathy. Patients with (vs. without) neck pain tended to be older, but, the difference was not statistically significant (Table 2). We found no statistical differences in gender and family history (inherited MTC, sporadic MTC, and sporadic MTC with somatic mutations) between the 2 groups. Similarly, we found no difference in preoperative ultrasound-proven lymphadenopathy between the 2 groups (Table 2 ).

Table I. Patients with medullary thyroid cancer (MTC) vs. papillary thyroid cancer (PTC)

\begin{tabular}{|c|c|c|c|}
\hline & MTC & PTC & p-value \\
\hline Total number of patients, $n$ & 50 & 50 & \\
\hline Age (years), mean \pm SD & $43.0 \pm 18.2$ & $49.9 \pm 16.3$ & 0.089 \\
\hline \multicolumn{4}{|l|}{ Gender, n (\%) } \\
\hline Female & $30(60)$ & $43(86)$ & \\
\hline Male & $20(40)$ & $7(14)$ & 0.006 \\
\hline \multicolumn{4}{|l|}{ Race, n (\%) } \\
\hline White & $34(68)$ & $29(58)$ & 0.408 \\
\hline Asian & $5(10)$ & $11(22)$ & 0.171 \\
\hline African American & $2(4)$ & $1(2)$ & 1.000 \\
\hline Latino & $2(4)$ & $1(2)$ & 1.000 \\
\hline Other & $7(14)$ & $8(16)$ & 1.000 \\
\hline \multicolumn{4}{|l|}{ Symptoms, n (\%) } \\
\hline Asymptomatic & $37(74)$ & $47(94)$ & 0.012 \\
\hline Neck pain & $11(22)$ & $3(6)$ & 0.041 \\
\hline Hoarseness & $2(4)$ & 0 & 0.495 \\
\hline \multicolumn{4}{|l|}{ Pathologic findings, $\mathrm{n}(\%)$} \\
\hline Multifocal & $26(52)$ & $27(54)$ & 1.000 \\
\hline Capsular invasion & $9(18)$ & $9(18)$ & 1.000 \\
\hline Vascular invasion & $5(10)$ & $2(4)$ & 0.437 \\
\hline Lymphatic invasion & $10(20)$ & $1(2)$ & 0.008 \\
\hline Lymph node (LN) metastases & $23(46)$ & $14(28)$ & 0.09 \\
\hline \multicolumn{4}{|l|}{ Stage of disease, $n(\%)$} \\
\hline Stage I & $16(32)$ & $36(72)$ & 0.0001 \\
\hline Stage II & $9(18)$ & $9(18)$ & 1.000 \\
\hline Stage III & $7(14)$ & $4(8)$ & 0.525 \\
\hline Stage IVa & $11(22)$ & $1(2)$ & 0.004 \\
\hline Stage IVb & 0 & 0 & 1.000 \\
\hline Stage IVc & $4(8)$ & 0 & 0.117 \\
\hline
\end{tabular}


Table 2. Patients with medullary thyroid cancer (MTC), with and without neck pain at presentation

\begin{tabular}{llll}
\hline Medullary thyroid cancer (MTC) & Pain & No pain & p-value \\
\hline Total number of patients, $\mathbf{n}$ & 11 & 39 & 0.073 \\
Age (years), mean \pm SD & $51.6 \pm 11.5$ & $40.6 \pm 19.1$ & \\
Gender, $\mathbf{n}(\%)$ & & & \\
Female & $7(64)$ & $23(59)$ & 1.000 \\
Male & $4(36)$ & $16(41)$ & 1.000 \\
Family history of MTC, $\mathbf{n}(\%)$ & $2(18)$ & $15(38)$ & 1.000 \\
Ultrasound-proven nodal disease, $\mathbf{n}(\%)$ & $2(18)$ & $6(15)$ & 0.340 \\
Histologic findings, $\mathbf{n}(\%)$ & & & 0.314 \\
Tumor diameter (cm), mean \pm SD & $2.2 \pm 1.4$ & $1.8 \pm 1.4$ & 1.000 \\
Multifocal & $4(36)$ & $22(56)$ & 0.301 \\
Capsular invasion & $2(18)$ & $7(18)$ & 0.424 \\
Vascular invasion & $2(18)$ & $3(8)$ & $\mathbf{0 . 0 1 4}$ \\
Lymphatic invasion & $1(9)$ & $9(23)$ & \\
Lymph node (LN) metastases & $9(82)$ & $14(36)$ & 0.466 \\
Stage of disease, $\mathbf{n}(\%)$ & & & 0.178 \\
Stage I & $2(18)$ & $14(36)$ & 0.641 \\
Stage II & 0 & $9(23)$ & $\mathbf{0 . 0 4 8}$ \\
Stage III & $2(18)$ & $5(13)$ & 1.000 \\
Stage IVa & $5(45)$ & $6(15)$ & 0.206 \\
Stage IVb & 0 & 0 & $\mathbf{0 . 0 1 4}$ \\
Stage IVc & $2(18)$ & $23(64)$ & \\
Stages I-II, grouped together & $2(18)$ & $13(36)$ & \\
Stages III-IV, grouped together & $9(82)$ & & \\
\hline
\end{tabular}

Table 3. Patients with papillary thyroid cancer (PTC), with and without neck pain at presentation

\begin{tabular}{llll}
\hline Papillary thyroid cancer (PTC) & Pain & No pain & p-value \\
\hline Total number of patients, $\mathbf{n}$ & 3 & 47 & 0.019 \\
Age (years), mean \pm SD & $73.7 \pm 9.9$ & $48.4 \pm 15.4$ & \\
Gender, $\mathbf{n}(\%)$ & & $40(85)$ & 1.000 \\
Female & $3(100)$ & $7(15)$ & 1.000 \\
Male & 0 & $2(4)$ & 0.347 \\
Family history of PTC & 0 & & 1.000 \\
Histologic findings, $\mathbf{n}(\%)$ & & $1.6 \pm 1.7$ & 0.456 \\
Tumor diameter (cm), mean \pm SD & $1.1 \pm 1.2$ & $25(53)$ & 1.000 \\
Multifocal & $2(67)$ & $8(17)$ & 1.000 \\
Capsular invasion & $1(33)$ & $2(4)$ & 1.000 \\
Vascular invasion & 0 & $1(2)$ & 1.000 \\
Lymphatic invasion & 0 & $13(28)$ & 0.456 \\
Lymph node (LN) metastases & $1(33)$ & & 1.000 \\
Stage of disease, $\mathbf{n}(\%)$ & & $34(72)$ & 1.000 \\
Stage I & $2(67)$ & $8(17)$ & 1.000 \\
Stage II & $1(33)$ & $4(9)$ & $1(2)$ \\
Stage III & & & \\
Stage IVa & & $42(89)$ & \\
Stage IVb & & $5(11)$ & \\
Stage IVc & $3(100)$ & & \\
Stages I-II, grouped together & & & \\
Stages III-IV, grouped together & & \\
\hline
\end{tabular}


No histologic differences per the final pathology test results were apparent between the 2 groups; the rate of multifocality, capsular invasion, vascular invasion, and lymphatic invasion were similar (Table 2). However, patients with neck pain were more likely to have LN metastases (82\%) than those without neck pain $(36 \%)$; of which 7 of $9(78 \%)$ patients with neck pain compared to 7 of $14(50 \%)$ without neck pain had both central and ipsilateral lateral neck LN metastases. This difference was statistically significant $(p=$ 0.014 ) and translated into a higher stage of disease. Of patients with neck pain, only $18 \%$ had either stage I or II disease, as compared with $64 \%$ without neck pain; however, $82 \%$ of patients with neck pain had stage III or IV disease, as compared with only $36 \%$ without pain $(p=0.014)$. In 3 patients without neck pain, we could not definitively determine the stage of disease.

Unlike in our MTC study group, the age of patients with vs. without neck pain differed in our PTC control group. Patients with PTC with (vs. without) neck pain were older (median age, 73.7 years with neck pain vs. 48.4 without, $\mathrm{p}=0.019$ ). As in our MTC study group, we found no difference in gender, genetic makeup, or histologic findings in our PTC control group (Table 3). We also found no difference in our PTC control group in the incidence of LN metastases between patients with vs. without neck pain, nor did the stage of disease differ in any way between those 2 subgroups of patients with PTC (Table 3).

\section{Discussion}

MTC, an aggressive cancer, is associated with a worse prognosis than WDTC of follicular cell origin.[5] In patients with MTC, the high incidence of LN metastases increases the risk of developing disseminated disease and contributes to their decreased survival rate. A cure can be achieved in most patients with MTC who do not have LN metastases, but the presence of LN metastases adversely affects the possibility of a biochemical cure and of survival.[6] Multifocal disease is associated with an increased risk of capsular and extrathyroidal invasion; the presence of LN metastases in patients with MTC and in patients with PTC portends a worse prognosis. Additionally, in both groups, multifocality is a predictor of LN metastases.[10] The presence of central LN metastases also increases the risk of lateral LN involvement.[11]

Despite our knowledge of histologic indicators of LN metastases, no good clinical predictors of LN metastases exist. In our study, we set out to determine (a) whether or not anterior neck pain at presentation was more common in patients with MTC than in those with PTC and (b) whether or not neck pain predicted LN metastases.
We found no statistical difference in the incidence of LN metastases between patients with MTC and patients with PTC (Table 1). However, patients with MTC had significantly more anterior neck pain, as compared with patients with patients with PTC. Moreover, patients with MTC with neck pain had a significantly higher risk of having LN metastases, as compared with their fellow patients with MTC without neck pain. This difference also translated into a higher rate of concurrent central and ipsilateral lateral LN metastases in our MTC pain group, compared to MTC patients without pain. But in our control group of patients with PTC, we found no significant difference between those with and without neck pain. The difference in the incidence of LN metastases between MTC patients with and without neck pain reflects the higher stage of disease in those with neck pain $(82 \%$ at stage III to IV), as compared with those without neck pain ( $36 \%$ at stages III to IV). This difference in the stage of disease was not apparent in our control group of patients with PTC (Table 3).

Our study has several limitations. First, although symptoms were documented prospectively, this is a retrospective analysis, which may have resulted in a selection bias in favor of patients with neck pain. Second, although we attempted to normalize our data by including a control group of an equal number of patients with PTC, our study group and our control group were not matched for the stage of disease. More patients with stage III or IV disease were in our study group (patients with MTC), as compared with our control group (patients with PTC). Although we found a statistically significant difference in the number of patients with anterior neck pain in our study (MTC) group, as compared with our control (PTC) group, this difference may simply reflect the larger number of patients with stage III or IV disease in our study (MTC) group - although we feel that is unlikely, because several patients had painful thyroid nodules for several years before treatment. Third, the small sample size of both our study group and our control group may not have ensured adequate representation of the general population. However, this limitation is a reflection of the rarity of MTC and the difficulty in obtaining a larger series at a single institution. Forth, although we found no difference in presentation between sporadic and inherited MTC, the small number of patients with inherited MTC may not have allowed adequate assessment. Lastly, since our institution is a tertiary referral hospital, we were unable to obtain all the calcitonin and CEA levels at the initial diagnosis of each patient. This limited our ability to correlate the biochemical levels with the symptom of anterior neck pain. 
In summary, the results of our study suggest that more patients with MTC present with anterior neck pain than do patients with PTC and that those with MTC have an increased risk of LN metastases (despite having no physical or ultrasound evidence of LN metastases), as compared with patients with PTC. Most clinicians agree that MTC is an aggressive disease and therefore a bilateral prophylactic central neck dissection should be performed at the time of total thyroidectomy according to the ATA guidelines.[5] However, reservations may exist about performing a bilateral central neck dissection in patients with sporadic MTC. This study confirms the aggressive nature of MTC, especially in patients who present with concomitant anterior neck pain. The results of this retrospective analysis support the ATA guidelines recommendation to perform a prophylactic central lymph node dissection in patients with MTC. Furthermore, our study suggests that MTC patients presenting with anterior neck pain should undergo a simultaneous ipsilateral lateral neck dissection at the initial operation. However, future prospective studies are needed to verify and support our findings.

\section{Acknowledgements}

We would like to thank the Friends of Endocrine, Helen and Sanford Diller Family Foundation, the Jerrold Heller Family Foundation, Dr. and Mrs. Andrew S. Grove, and the Bell Charitable Foundation for their support.

We would also like to thank Mary E. Knatterud, $\mathrm{PhD}$ for her help in editing and revising this manuscript.

\section{Conflict of Interest}

The authors have declared that no conflict of interest exists.

\section{References}

1. Roman S, Mehta P, Sosa JA. Medullary thyroid cancer: early detection and novel treatments. Curr Opin Oncol 2009;21:5-10

2. Scollo C, Baudin E, Travagli JP, et al. Rationale for central and bilateral lymph node dissection in sporadic and hereditary medullary thyroid cancer. J Clin Endocrinol Metab 2003;88:2070-2075

3. Jimenez C, Hu MI, Gagel RF. Management of medullary thyroid carcinoma. Endocrinol Metab Clin North Am 2008;37:481-496

4. de Groot JW, Plukker JT, Wolffenbuttel BH, et al. Determinants of life expectancy in medullary thyroid cancer: age does not matter. Clin Endocrinol (Oxf) 2006;65:729-736

5. Kloos RT, Eng C, Evans DB, et al. Medullary thyroid cancer: management guidelines of the American Thyroid Association. Thyroid 2009;19:565-612

6. Boostrom SY, Grant CS, Thompson GB, et al. Need for a revised staging consensus in medullary thyroid carcinoma. Arch Surg 2009;144:663-669
7. Gülben K, Berberoğlu U, Boyabatli M. Prognostic factors for sporadic medullary thyroid carcinoma. World Journal of Surgery 2006;30:84-90

8. Moley JF, Fialkowski EA. Evidence-based approach to the management of sporadic medullary thyroid carcinoma. World Journal of Surgery 2007;31:946-956

9. Panigrahi B, Roman SA, Sosa JA. Medullary Thyroid Cancer: Are Practice Patterns in the United States Discordant From American Thyroid Association Guidelines? Ann Surg Oncol 2010;17:1490-1498

10. Machens A, Hauptmann S, Dralle H. Increased risk of lymph node metastasis in multifocal hereditary and sporadic medullary thyroid cancer. World Journal of Surgery 2007;31:1960-1965

11. Machens A, Hauptmann S, Dralle H. Prediction of lateral lymph node metastases in medullary thyroid cancer. The British Journal of Surgery 2008;95:586-591 